\title{
Fetal cardiac intervention: Improved results of fetal cardiac bypass in immature fetuses using the TinyPump device
}

\author{
Vinod A. Sebastian, MD, ${ }^{\text {a }}$ Giuseppe Ferro, MD, ${ }^{\text {a }}$ Hiroshi Kagawa, MD, PhD, ${ }^{\text {a }}$ Teimour Nasirov, MD, ${ }^{a}$ \\ Katsuhide Maeda, MD, PhD, ${ }^{\mathrm{a}}$ William T. Ferrier, MS, DVM, ${ }^{\mathrm{b}}$ Setsuo Takatani, PhD, DMed, ${ }^{\mathrm{c}}$ \\ R. Kirk Riemer, PhD, ${ }^{a}$ Frank L. Hanley, MD, ${ }^{a}$ and V. Mohan Reddy, MD ${ }^{\mathrm{a}}$
}

\begin{abstract}
Objective: Fetal cardiac surgery is a potential innovative treatment for certain congenital heart defects that have significant mortality and morbidity in utero or after birth, but it has been limited by placental dysfunction after fetal cardiac bypass. We have used the TinyPump device for fetal cardiac bypass in sheep fetuses at 90 to 110 days gestation.
\end{abstract}

\begin{abstract}
Methods: Ten mixed-breed pregnant ewes were used over a period of 6 months, and 10 fetuses were placed on bypass for 30 minutes. Five fetuses with a mean gestational age of $104 \pm 4.5$ days and mean weight of $1.4 \pm 0.4$ $\mathrm{kg}$ were placed on bypass using the TinyPump device, and 5 fetuses with a mean gestational age of $119 \pm 4.5$ days and mean weight of $3.4 \pm 0.4 \mathrm{~kg}$ were placed on bypass using the roller head pump. The fetuses were monitored for up to 3 hours after bypass or until earlier demise.
\end{abstract}

Results: Progressive respiratory and metabolic acidosis developed in all fetuses. The TinyPump group had a lower gestational age and weight compared with the roller head pump group. However, the rate of postbypass deterioration in the TinyPump group, as measured with blood gases, was noted to be significantly slower compared with the roller head pump group.

Conclusions: We demonstrate the feasibility of the TinyPump device for fetal cardiac bypass in a fetal sheep model. The TinyPump group showed improved results compared with the roller head group despite more immature fetuses. The TinyPump device seems to be a promising device for future studies of fetal cardiac bypass in immature fetal sheep and in primates. (J Thorac Cardiovasc Surg 2013;145:1460-4)

Fetal cardiac surgery is an exciting potential innovative therapy for the treatment of complex cardiac anomalies that start out as relatively simple lesions in early gestation. Fetal echocardiography can permit the diagnosis of congenital heart defects during early gestation. Some complex congenital cardiac lesions are thought to be the result of relatively simple primary lesions that progress because of altered intracardiac blood flow patterns ${ }^{1}$ that may be altered with fetal therapy. Most open fetal cardiac interventions will require some form of fetal extracorporeal circulation (fetal cardiac bypass). The feasibility of fetal cardiac bypass has been shown, and the physiologic consequences of fetal cardiac bypass, including severe placental dysfunction, have been described. ${ }^{2-9}$ Stimuli likely responsible for placental dysfunction are

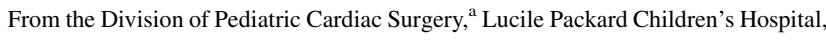
Stanford University School of Medicine, Stanford, Calif; Department of Medicine and Epidemiology, ${ }^{\mathrm{b}}$ University of California, Davis, School of Veterinary Medicine, Davis, Calif; and Department of Artificial Organs, ${ }^{c}$ Tokyo Medical and Dental University, Tokyo, Japan.

Disclosures: Authors have nothing to disclose with regard to commercial support.

Abstract read at the American Academy of Pediatrics, National Conference and Exhibition, Boston, Massachusetts, October 15-18, 2011.

Received for publication May 26, 2012; revisions received July 19, 2012; accepted for publication Aug 1, 2012; available ahead of print Sept 3, 2012.

Address for reprints: Vinod A. Sebastian, MD, Division of Pediatric Cardiac Surgery, 300 Pasteur Dr, CVRB MC 5407, Stanford, CA 94305 (E-mail: vinodas@yahoo. com).

0022-5223/\$36.00

Copyright (c) 2013 by The American Association for Thoracic Surgery

http://dx.doi.org/10.1016/j.jtcvs.2012.08.014 extracorporeal surfaces, priming substances, flow characteristics, and fetal stress. Large cardiac bypass circuits also require adult blood for priming, which has a different oxygen affinity than fetal blood. We previously showed that the use of the Hemopump (Modified model of HP24 sternotomy pump; Johnson \& Johnson Interventional Systems, Rancho Cordova, Calif) to reduce extracorporeal surface area and avoid external priming substances leads to improved placental hemodynamics after fetal cardiac bypass. ${ }^{10}$ The Hemopump currently is not in production, and its minimal flow rate was too high when used in primate fetuses and lamb fetuses weighing less than $1500 \mathrm{~g}$ for fetal cardiac bypass.

The TinyPump device is a disposable centrifugal pump that was developed at Tokyo Medical and Dental University in 2004. ${ }^{11}$ The TinyPump device comprises a console, reusable motor driver, and disposable pump head easily attached to and detached from the motor driver. Six pole magnets are installed inside the impeller and radially coupled to the outer driver magnets rotated by a direct current motor. The impeller motor is suspended approximately $1 \mathrm{~mm}$ above the bottom surface of the housing by a radial magnetic coupling force and further suspended by a hydrodynamic bearing at the center of the impeller, creating an effect similar to a noncontact suspension of the impeller. A secondary flow inside the pump head is designed to pass through between the impeller and the 
bottom housing to acquire a better washout effect and to possibly prevent thrombus formation inside the pump head. The pump has $5 \mathrm{~mL}$ of priming volume including the space inside the one-quarter inch inflow and outflow ports. It weighs $150 \mathrm{~g}$, including a reusable motor drive and measures $49 \times 20 \mathrm{~mm}$. The impeller rotation supported by the hydrodynamic bearing at the center can achieve speeds between 500 and $5000 \mathrm{rpm}$. When used with onequarter inch inflow and outflow cannulae, the pump is capable of providing flow of 0.1 to $4.0 \mathrm{~L} / \mathrm{min}$ against a head pressure of $100 \mathrm{~mm} \mathrm{Hg}$ at pump speeds of 1500 to $4500 \mathrm{rpm}$.

In the fetal sheep model using the TinyPump device, we have been able to obtain flow rates from 10 to $1000 \mathrm{~mL} / \mathrm{min}$, which has been adequate in our model and weight range. This ability to provide a wide range of flows at both the lower and upper ranges is crucial in advancing the fetal cardiac bypass model to earlier stages of gestation and to primate models that have smaller fetuses.

\section{MATERIALS AND METHODS \\ Extracorporeal Circuits}

TinyPump circuit. The circuit is shown in Figure 1. The circuit uses the TinyPump device, which has inflow and outflow connectors for onequarter inch tubing and has a priming volume of $5 \mathrm{~mL}$. The circuit has a priming volume of $10 \mathrm{~mL}$ and 1 to 2 three-way stopcocks that can be incorporated for deairing purposes. The circuit is primed with normosol (Abbott Laboratories, Abbott Park, Ill), and the cannulae are backbled with fetal blood. The small amount of normosol used for priming does not seem to cause any significant hemodilution.

Conventional circuit. The conventional circuit consists of polyvinyl one-quarter inch tubing, a collapsible neonatal extracorporeal membrane oxygenation reservoir (mounted on plexiglass to prevent kinking), a roller head pump (Cole-Parmer, Vernon Hills, Ill), and an in-line flow probe (Transonic Systems, Inc, Ithaca, NY) (Figure 1). The circuit priming volume varies from 75 to $100 \mathrm{~mL}$ and is primed with fresh adult sheep venous blood.

\section{Fetal Cardiac Bypass}

The ewes were fasted overnight before the study and received cefazolin intravenously $(20 \mathrm{mg} / \mathrm{kg})$ preoperatively and during the procedure. They were premedicated with ketamine/valium intravenously. The ewe was then placed supine on the operating table, intubated, and placed under isoflurane maintenance anesthesia. The ewes were ventilated with a Hallowell Veterinary Model 2000 ventilator (Hallowell Engineering \& Manufacturing Corp, Pittsfield, Mass), and capnography was used in addition to blood gases for assessment of ventilation. A central venous catheter and arterial line were placed in the maternal jugular vein and carotid artery, respectively, for monitoring. Maternal heart rate, blood pressure, central venous pressure, and blood gases were monitored, and lactated Ringer's solution was given at 5 to $10 \mathrm{~mL} / \mathrm{kg} / \mathrm{h}$. A midline laparotomy was performed, and the uterus was exposed. The number and orientation of fetuses were determined by palpation.

The fetal chest was palpated, and a hysterotomy was made directly over the fetal chest, taking care to avoid the multiple cotyledons of the sheep placenta. The fetal jugular vein and carotid artery were cannulated for venous access and arterial blood pressure monitoring. Normosol infusion at $1 \mathrm{~mL} / \mathrm{kg} / \mathrm{min}$ was started, and after the bypass protocol, dopamine at $5 \mu \mathrm{g} / \mathrm{kg} / \mathrm{min}$, epinephrine at $0.05 \mu \mathrm{g} / \mathrm{kg} / \mathrm{min}$, and calcium chloride at $20 \mathrm{mg} / \mathrm{kg} /$ hour were administered.
A midline fetal sternotomy and pericardiotomy were performed, and the heart was exposed. The main pulmonary artery and superior vena cava were dissected. Purse-string sutures were placed in the main pulmonary artery and superior vena cava for arterial and venous cannulation, respectively. The fetus was anticoagulated with $300 \mathrm{U} / \mathrm{kg}$ of heparin sodium given through the jugular central line. A $6 \mathrm{~F}$ to $10 \mathrm{~F}$ arterial cannula was used for arterial cannulation. The right atrium was cannulated through the superior vena cava because of the friable nature of fetal sheep atrium, and $8 \mathrm{~F}$ to $12 \mathrm{~F}$ venous cannulae were used. In the TinyPump group, the bypass circuit was primed with $10 \mathrm{~mL}$ of normosol and backbleeding of fetal blood. In the conventional roller head group, the bypass circuit was primed with adult blood. After deairing maneuvers were performed, normothermic beating heart cardiac bypass was performed for 30 minutes using the placenta as the sole oxygenator.

Pump flows were maintained at the maximum achievable flow in any given fetus with an intention to provide at least $200 \mathrm{~mL} / \mathrm{kg} / \mathrm{min}$ of flow. This is based on previous research showing that high flows are required in fetal cardiac bypass when the placenta is used as the oxygenator. ${ }^{12,13}$ Pump flows were continuously monitored with the in-line flow probe After bypass discontinuation, infusion of dopamine at $5 \mu \mathrm{g} / \mathrm{kg} / \mathrm{min}$, epinephrine at $0.05 \mu \mathrm{g} / \mathrm{kg} / \mathrm{min}$, and calcium chloride at $20 \mathrm{mg} / \mathrm{kg} / \mathrm{hour}$ was initiated. The fetus was monitored for up to 180 minutes or until earlier demise. All baseline maternal and fetal hemodynamic data (maternal and fetal heart rates, maternal and fetal central venous pressures, maternal and fetal arterial pressures) were continuously recorded. Baseline maternal and fetal blood gases were measured. Fetal blood gases were monitored every 15 minutes during bypass and every 30 minutes in the postbypass period. After completion of the study, the ewes and fetuses were euthanized. The dead fetuses were delivered through the hysterotomy, towel-dried, and weighed.

All animals received humane care in compliance with the 1996 Guide for the Care and Use of Laboratory Animals, recommended by the US National Institutes of Health. The experimental protocol was approved by the Institutional Animal Care and Use Committees at Stanford and at the University of California, Davis.

\section{Data Acquisition}

Fetal and maternal systemic arterial pressures and fetal and maternal central venous pressures were measured using Statham P23Db pressure transducers (Statham Instruments, Inc, Hato Rey, Puerto Rico). Mean pressures were obtained by electric integration. Heart rate was measured by a cardiotachometer triggered from the phasic systemic arterial pressure pulse wave. All flows were measured on an ultrasonic flowmeter (Transonic Systems Inc). Fetal and maternal blood gases were measured using an ABL 800 blood gas analyzer (Radiometer America, Westlake, Ohio).

\section{RESULTS}

Ten mixed-breed pregnant ewes were used over a period of 6 months, and 10 fetuses were placed on bypass for 30 minutes. Five fetuses with a mean gestational age of $104 \pm 4.5$ days and mean weight of $1.4 \pm 0.4 \mathrm{~kg}$ were placed on bypass using the TinyPump device, and 5 fetuses with a mean gestational age of $119 \pm 4.5$ days and a mean weight of $3.4 \pm 0.4 \mathrm{~kg}$ were placed on bypass using the roller head pump. All fetuses developed progressive respiratory and metabolic acidosis. However, the rate of postbypass deterioration in the TinyPump group, as measured with blood gases, was noted to be significantly slower compared with the roller head pump group.

The mean gestational age, mean fetal weight, arterial blood gas data, and peak flow rate obtained in the TinyPump 

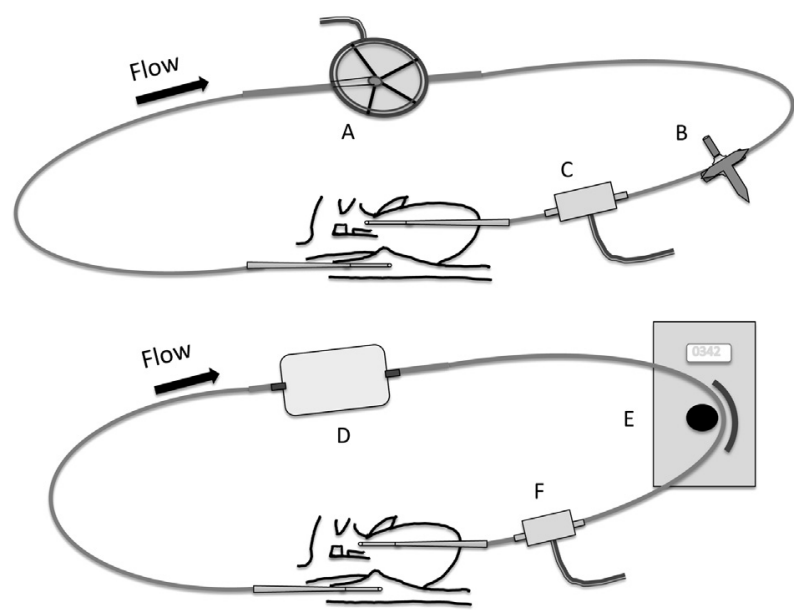

FIGURE 1. TinyPump circuit comprising the TinyPump (A), 3-way stop cock for deairing (B), and flow probe (C). Priming volume of circuit is $10 \mathrm{~mL}$. Conventional fetal cardiac bypass circuit comprising venous reservoir (D), roller head pump (E), and flow probe (F). Priming volume of the circuit varies from 75 to $100 \mathrm{~mL}$.

and roller head groups were compared using the modified Student $t$ test. The roller head pump group of fetuses had a higher gestational age and weight compared with the TinyPump fetuses (Figure 2). The roller head group had a mean gestational age of $119 \pm 4.5$ days $(\mathrm{n}=5$, $P=.04$ ) versus $104 \pm 4.5$ days in the TinyPump group. The mean weight of the roller head pump fetuses was 3.4 $\pm 0.4 \mathrm{~kg}$ versus $1.4 \pm 0.4 \mathrm{~kg}$ in the TinyPump group $(\mathrm{n}=5$, $P=.008)$. Despite this inherent disadvantage in gestational age and weight, the TinyPump group had more favorable blood gas results than the roller head pump group.

\section{Arterial Blood Gases}

Table 1 shows the blood gases obtained in the 2 groups of fetuses that had the roller head pump and TinyPump used for fetal cardiac bypass. We found statistically significant improvement in carbon dioxide tension and lactate levels in the TinyPump group (Figure 3 and Table 1).

\section{Peak Flow Rate Obtained}

We noticed a significantly higher peak flow rate in the TinyPump group compared with the roller head group (Figure 4). The peak flow rate obtained in the TinyPump group was $236 \pm 19 \mathrm{~mL} / \mathrm{kg} / \mathrm{min}(\mathrm{n}=5)$ versus $124 \pm$ $19 \mathrm{~mL} / \mathrm{kg} / \mathrm{min}(\mathrm{n}=5)(P=.003)$.

\section{DISCUSSION}

The flow-related theory of cardiac morphogenesis and the earlier prenatal echocardiographic detection of congenital cardiac disease suggest that in utero repair of certain congenital cardiac diseases, such as pulmonary atresia with intact ventricular septum, critical aortic stenosis, tetralogy with absent pulmonary valve syndrome, and
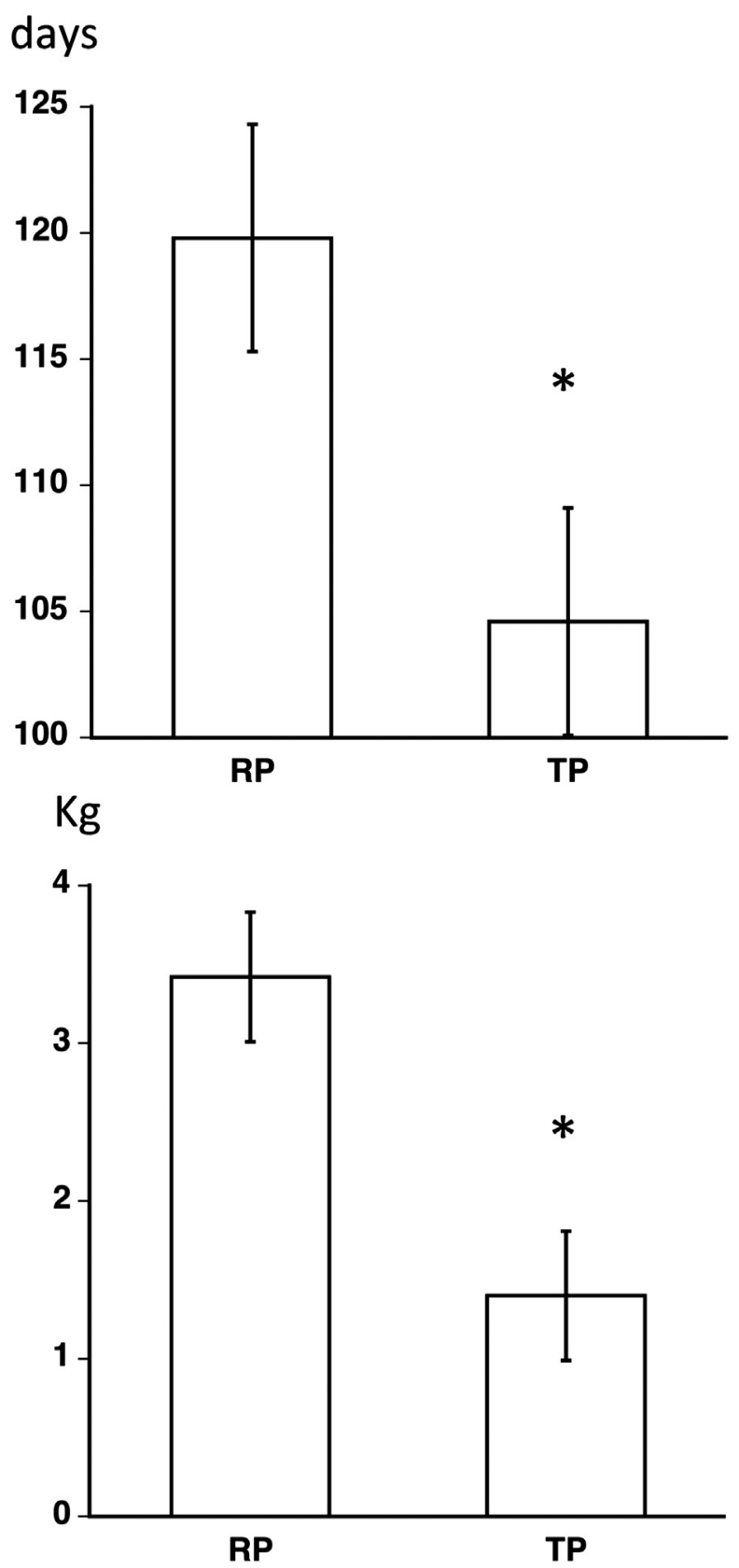

FIGURE 2. Comparison between gestational age and body weight of the roller head pump group versus the TinyPump group showed younger gestational age and lower body weight in the TinyPump group: gestational age 119 days versus $104 \pm 4.5$ days $(\mathrm{n}=5, P=.04)$ and body weight 3.4 $\mathrm{kg}$ versus $1.4 \pm 0.4 \mathrm{~kg}(\mathrm{n}=5, P=.008)$. *Statistically significant difference in gestational age and body weight. $R P$, Roller pump; $T P$, TinyPump.

severe Ebstein's anomaly, may be highly advantageous. Most of these interventions will require the use of fetal cardiac bypass, which has the limitation of producing placental dysfunction.

The clinical application of fetal noncardiac surgery and catheter-based cardiac intervention has been carried out 
TABLE 1. Blood gas values in TinyPump and roller head pump groups at 30, 60, and 90 minutes after fetal cardiac bypass

\begin{tabular}{lllcc}
\hline & Type of pump & \multicolumn{1}{c}{ 30 $\mathbf{m i n}$ after bypass } & 60 min after bypass & 90 min after bypass \\
\hline Mean pH & Roller head pump & $7.12 \pm 0.04(\mathrm{n}=5)$ & $7.02 \pm 0.06(\mathrm{n}=5)$ & $7.04 \pm 0.09(\mathrm{n}=3)$ \\
& TinyPump & $7.18 \pm 0.04(\mathrm{n}=5)(P=.8)$ & $7.15 \pm 0.07(\mathrm{n}=4)(P=.9)$ & $7.07 \pm 0.08(\mathrm{n}=4)(P=.5)$ \\
${\mathrm{Mean} \mathrm{PO}_{2}}$ & Roller head pump & $21.5 \pm 4(\mathrm{n}=5)$ & $21.6 \pm 3.9(\mathrm{n}=5)$ & $24.8 \pm 6(\mathrm{n}=3)$ \\
& TinyPump & $26.8 \pm 4(\mathrm{n}=5)(P=.8)$ & $18.7 \pm 4.3(\mathrm{n}=4)(P=.3)$ & $17.5 \pm 5.2(\mathrm{n}=4)(P=.2)$ \\
Mean $\mathrm{PCO}_{2}$ & Roller head pump & $\mathbf{8 9 . 1} \pm \mathbf{4 . 8}(\mathbf{n}=\mathbf{5})$ & $\mathbf{1 0 9 . 6} \pm \mathbf{1 1}(\mathbf{n}=\mathbf{5})$ & $\mathbf{1 1 4 . 2 3} \pm \mathbf{1 5 . 5}(\mathbf{n}=\mathbf{3})$ \\
& TinyPump & $\mathbf{5 7 . 8} \pm \mathbf{4 . 8}(\mathbf{n}=\mathbf{5})(\boldsymbol{P}=\mathbf{. 0 0 0 9})$ & $\mathbf{6 7 . 9} \pm \mathbf{1 2 . 3}(\mathbf{n}=\mathbf{4})(\boldsymbol{P}=\mathbf{. 0 2})$ & $\mathbf{7 6 . 9} \pm \mathbf{1 3 . 4}(\mathbf{n}=\mathbf{4})(\boldsymbol{P}=\mathbf{. 0 0 9})$ \\
Mean lactate & Roller head pump & $\mathbf{8 . 2} \pm \mathbf{1 . 1 6}(\mathbf{n}=\mathbf{5})$ & $\mathbf{1 0 . 5} \pm \mathbf{1 . 1}(\mathbf{n}=\mathbf{5})$ & $\mathbf{1 1 . 4} \pm \mathbf{1 . 3}(\mathbf{n}=\mathbf{3})$ \\
& TinyPump & $\mathbf{3 . 7} \pm \mathbf{1 . 1 6}(\mathbf{n}=\mathbf{5})(\boldsymbol{P}=\mathbf{. 0 1})$ & $\mathbf{3 . 9} \pm \mathbf{1 . 2 5}(\mathbf{n}=\mathbf{4})(\boldsymbol{P}=\mathbf{. 0 0 3})$ & $\mathbf{5 . 4} \pm \mathbf{1 . 1 6}(\mathbf{n}=\mathbf{4})(\boldsymbol{P}=\mathbf{. 0 0 9})$ \\
\hline
\end{tabular}

Statistically significant data are in bold. $\mathrm{PO}_{2}$, Oxygen tension; $\mathrm{PCO}_{2}$, carbon dioxide tension.

mostly at approximately 24 to 29 weeks human gestation or earlier. This corresponds to 90 to 110 days sheep gestation. $^{14}$ The use of sheep fetuses at earlier gestation is more relevant to current clinical attempts at fetal therapy. Immature fetuses may have different uteroplacental hemodynamics and seems to be more susceptible to placental dysfunction than more mature fetuses. Placental dysfunction continued to be a major obstacle in these immature fetuses, and although the rate of decline of placental dysfunction was noted to be slower than in mature fetuses undergoing bypass with the roller head pump, we generally could not prevent the ultimate deterioration of placental

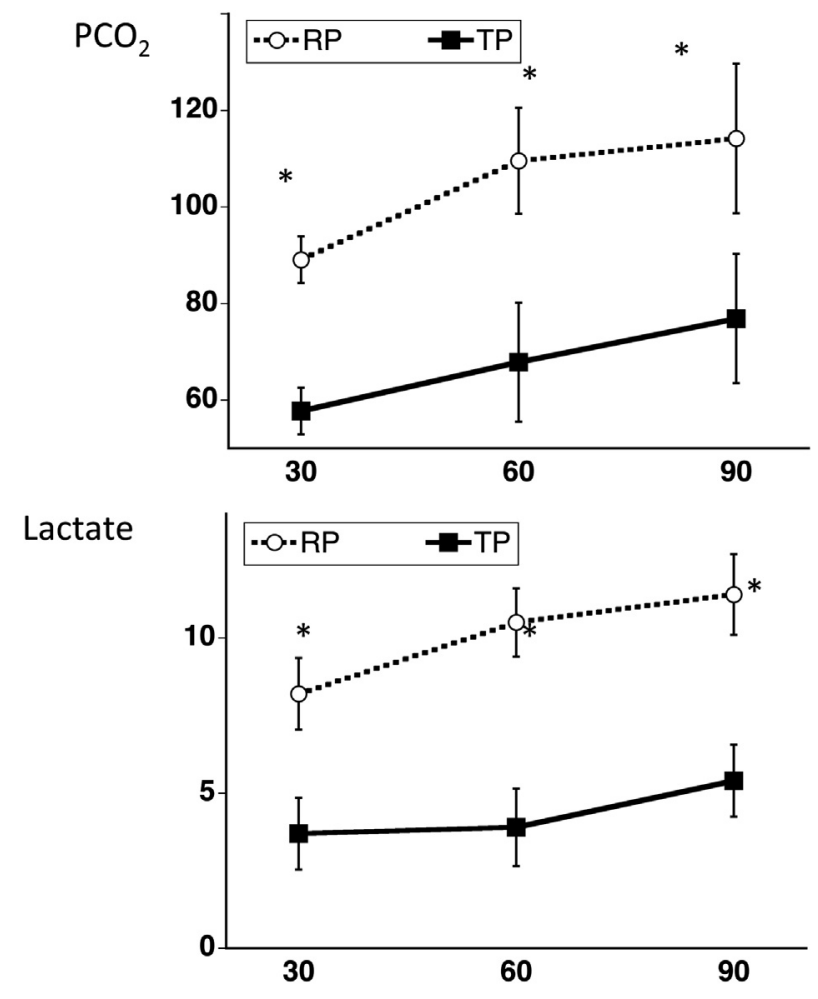

FIGURE 3. Comparison between carbon dioxide tension $\left(\mathrm{PCO}_{2}\right)$ and lactate in the roller head pump and TinyPump groups showed significantly higher $\mathrm{PCO}_{2}$ and lactate values in the roller head pump group at 30,60, and 90 minutes after fetal cardiac bypass. *Statistically significant difference in $\mathrm{PCO}_{2}$ and lactate levels. $\mathrm{PCO}_{2}$, Carbon dioxide tension; $R P$, roller pump; $T P$, TinyPump. function. Technical limitations in terms of tissue fragility and size of cannulae to be used are even more relevant in this gestational age than in the mature fetus. Even small amounts of blood loss during cannulation may result in significantly lower hematocrit, and fluid resuscitation leads to hemodilution more easily than in the mature fetus. All these issues require further work to elucidate techniques of fetal cardiac bypass at this gestational age.

These immature sheep fetuses are closer in size to primate fetuses, although primate fetal tissue may have higher tensile strength than sheep fetuses. Pump flow rate during bypass is also a critical parameter for maintenance of fetal gas exchange. In our previous primate experiments using the roller head pump, we had to infuse crystalloid at $50 \mathrm{~mL} / \mathrm{kg} / \mathrm{min}$ to maintain an adequate bypass flow (maximum flow of $176 \pm 70 \mathrm{~mL} / \mathrm{kg} / \mathrm{min}$ ) and hemodynamics. ${ }^{15}$ This was done using a $10 \mathrm{~F}$ atrial venous cannula and $14-$ or 16-gauge arterial cannula. In the present study and in

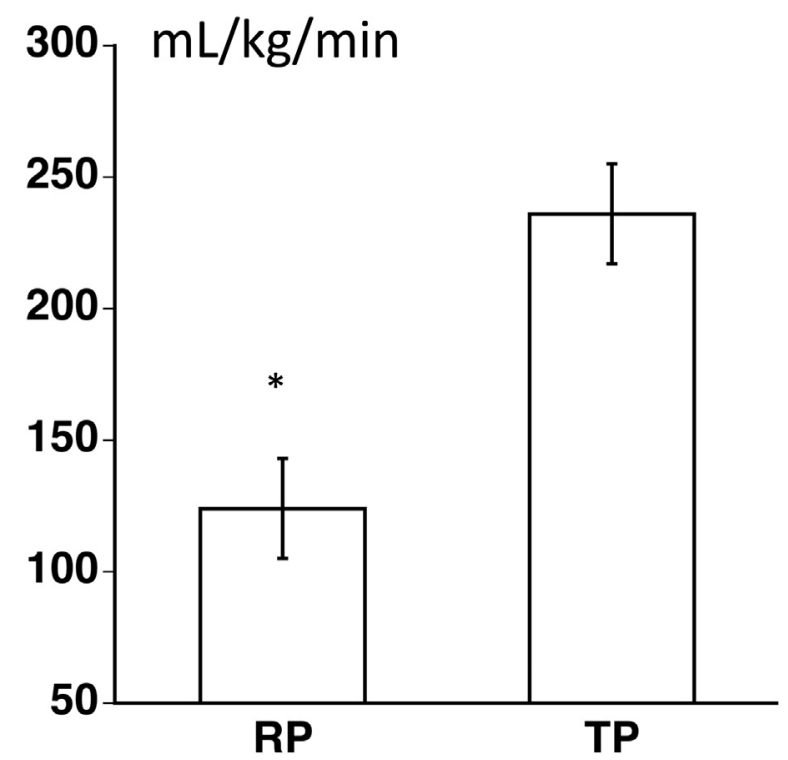

FIGURE 4. Comparison between peak flow rate in the roller head pump and TinyPump groups showed a higher peak flow rate in the TinyPump group: $236 \mathrm{~mL} / \mathrm{kg} / \mathrm{min}$ versus $124 \pm 19 \mathrm{~mL} / \mathrm{kg} / \mathrm{min}(\mathrm{n}=5, P=.003)$. *Statistically significant difference in peak flow rate. $R P$, Roller pump; $T P, \mathrm{Ti}-$ nyPump. 
pilot studies in primates using the TinyPump device, we did not require these extremely high amounts of intravenous fluid resuscitation. We have followed the guidelines recommended by Baker and colleagues ${ }^{16}$ for intravenous fluid resuscitation at $1 \mathrm{~mL} / \mathrm{kg} / \mathrm{h}$. Peak flows of $236 \mathrm{~mL} / \mathrm{kg} / \mathrm{min}$ were obtained using $10 \mathrm{~F}$ to $12 \mathrm{~F}$ venous cannulae (inserted through the superior vena cava) and $6 \mathrm{~F}$ to $8 \mathrm{~F}$ arterial cannula. The techniques used in fetal cardiac bypass in these immature sheep fetuses are more applicable to the primate model, which is an important step before any human application of fetal cardiac bypass. We anticipate that in primate models permitting direct atrial cannulation, the peak flows obtained during bypass will be even higher.

Our previous circuit with the Hemopump did not have any means to protect against air embolism. In the present circuit, we did not incorporate any bubble traps or air bubble detectors, but we did place 3 way stopcocks on both the venous and arterial side for manual deairing.

We have previously performed studies ${ }^{2-4}$ to block placental dysfunction in more mature fetuses. We are continuing to incorporate this pharmacologic blockage in the new TinyPump model.

\section{CONCLUSIONS}

We report the use of the TinyPump for fetal cardiac bypass in a fetal sheep model of 105 days gestational age. The pump showed improved results over the roller head group with improved blood gas parameters despite more immature fetuses in the TinyPump group. The pump has low priming volumes and a wide range of flow rates that can be easily adjusted. Both these factors make it a promising device for future studies of fetal cardiac bypass in immature fetal sheep and in primates, both of which are crucial before this therapy can be applied to humans.

\section{References}

1. Rychik J. Frontiers in fetal cardiovascular disease. Pediatr Clin North Am. 2004; 51:1489-502. vii.

2. Sabik JF, Heinemann MK, Assad RS, Hanley FL. High steroids prevent placental dysfunction after fetal cardiac bypass. J Thorac Cardiovasc Surg. 1994;107:116-25.

3. Fenton KN, Heinemann MK, Klautz RJM, Liddicoat JR, Hanley FL. Inhibition of fetal stress response improves cardiac output and gas exchange after fetal cardiac bypass. J Thorac Cardiovasc Surg. 1994;107:1416-22.

4. Sabik JF, Assad RS, Hanley FL. Prostaglandin synthesis inhibition prevents placental dysfunction after fetal cardiac bypass. J Thorac Cardiovasc Surg. 1994;103:733-42.

5. Assad RS, Lee F-Y, Bergner K, Hanley FL. Extracorporeal circulation in the isolated in situ lamb placental: hemodynamic characteristics. J Appl Physiol. 1992; 72:2176-80.

6. Assad RS, Lee F-Y, Sabik JF, Mackenzie S, Hanley FL. Tolerance of placenta to normothermic circulatory arrest. J Matern Fetal Invest. 1992;2: 145-50.

7. Fenton KN, Heinemann MK, Hanley FL. Exclusion of placenta during fetal cardiac bypass augments systemic flow and provides important information about the mechanism of placental injury. J Thorac Cardiovasc Surg. 1993;105: 502-12.

8. Hanley FL. Fetal responses to extracorporeal circulatory support. Cardiol Young. 1993;3:263-72.

9. Champsaur G, Parisot P, Martinot S, Ninet J, Robin J, Ovize M, et al. Pulsatility improves hemodynamics during fetal bypass: experimental study of pulsatile versus steady flow. Circulation. 1994;90(Suppl I):47-50.

10. Reddy VM, Liddicoat JR, Klein JR, McElhinney DB, Wampler RK, Hanley FL. Fetal cardiac bypass using an in-line axial flow pump to minimize extracorporeal surface and avoid priming volume. Ann Thorac Surg. 1996;62:393-400.

11. Takatani S, Hoshi H, Tajima K, Ohuchi K, Nakamura M, Asama J, et al. Feasibility of a miniature centrifugal rotary blood pump for low-flow circulation in children and infants. ASAIO J. 2005;51:557-62.

12. Hanley FL. Fetal cardiac surgery. In: Karp RB, Laks H, Wechsler AS, eds. Advances in Cardiac Surgery. Vol. 5. New York: Mosby-Year Book; 1994. p. $47-74$.

13. Hawkins JA, Clark SM, Shaddy RE, Gay WA Jr. Fetal cardiac bypass: improved placental function with moderately high flow rates. Ann Thorac Surg. 1994;52: 506-13.

14. Lombardi J, Sedgwick J, Schenbeck J, Lubbers W, Ferguson RE, Gardner A, et al. Cardiopulmonary bypass in the immature fetus through novel use of a mini-centrifugal pump. Perfusion. 2006;21:185-91.

15. Ikai A, Riemer RK, Ramamoorthy C, Malhotra S, Cassorla L, Amir G, et al. Preliminary results of fetal cardiac bypass in nonhuman primates. $J$ Thorac Cardiovasc Surg. 2005;129:175-81.

16. Baker RS, Lam CT, Heeb EA, Eghtesady P. Dynamic fluid shifts induced by fetal bypass. J Thorac Cardiovasc Surg. 2009;137:714-22. 